Research Article

\title{
Coefficient Bounds for Certain Subclasses of Analytic Functions Defined by Komatu Integral Operator
}

\author{
Serap Bulut \\ School of Civil Aviation College, Kocaeli University, Arslanbey Campus, 41285 Izmit-Kocaeli, Turkey \\ Correspondence should be addressed to Serap Bulut, serap.bulut@kocaeli.edu.tr \\ Received 23 March 2012; Accepted 11 July 2012 \\ Academic Editor: Yuri Latushkin \\ Copyright (c) 2012 Serap Bulut. This is an open access article distributed under the Creative \\ Commons Attribution License, which permits unrestricted use, distribution, and reproduction in \\ any medium, provided the original work is properly cited. \\ We determine the coeffcient bounds for functions in certain subclasses of analytic functions of \\ complex order, which are introduced here by means of a certain non-homogeneous Cauchy-Euler \\ type differential equation of order $m$. Relevant connections of some of the results obtained with \\ those in earlier works are also provided.
}

\section{Introduction, Definitions and Preliminaries}

Let $\mathbb{R}=(-\infty, \infty)$ be the set of real numbers, let $\mathbb{C}$ be the set of complex numbers,

$$
\mathbb{N}:=\{1,2,3, \ldots\}=\mathbb{N}_{0} \backslash\{0\}
$$

be the set of positive integers and

$$
\mathbb{N}^{*}:=\mathbb{N} \backslash\{1\}=\{2,3,4, \ldots\}
$$

Let $\mathcal{A}$ denote the class of functions of the form

$$
f(z)=z+\sum_{n=2}^{\infty} a_{n} z^{n}
$$


which are analytic in the unit disk:

$$
\mathbb{U}=\{z \in \mathbb{C}:|z|<1\} .
$$

Recently, Komatu [1] introduced a certain integral operator $L_{a}^{\delta}$ defined by

$$
L_{a}^{\delta} f(z)=\frac{a^{\delta}}{\Gamma(\delta)} \int_{0}^{1} t^{a-2}\left(\log \frac{1}{t}\right)^{\delta-1} f(z t) d t, \quad z \in \mathbb{U} ; a>0 ; \delta \geq 0 ; f(z) \in \mathcal{A} .
$$

Thus, if $f \in \mathcal{A}$ is of the form (1.3), then it is easily seen from (1.5) that (see [1])

$$
L_{a}^{\delta} f(z)=z+\sum_{n=2}^{\infty}\left(\frac{a}{a+n-1}\right)^{\delta} a_{n} z^{n}, \quad a>0 ; \delta \geq 0
$$

Using the relation (1.6), it is easily verfied that

$$
\begin{gathered}
z\left(L_{a}^{\delta+1} f(z)\right)^{\prime}=a L_{a}^{\delta} f(z)-(a-1) L_{a}^{\delta+1} f(z), \\
L_{a}^{\delta}\left(z f^{\prime}(z)\right)=z\left(L_{a}^{\delta} f(z)\right)^{\prime}
\end{gathered}
$$

We note that:

(i) for $a=1$ and $\delta=k$ ( $k$ is any integer), the multiplier transformation $L_{1}^{k} f(z)=I^{k} f(z)$ was studied by Flett [2] and Sălageăn [3];

(ii) for $a=1$ and $\delta=-k\left(k \in \mathbb{N}_{0}\right)$, the differential operator $L_{1}^{-k} f(z)=D^{k} f(z)$ was studied by Sălageăn [3];

(iii) for $a=2$ and $\delta=k$ ( $k$ is any integer), the operator $L_{2}^{k} f(z)=L^{k} f(z)$ was studied by Uralegaddi and Somanatha [4];

(iv) for $a=2$, the multiplier transformation $L_{2}^{\delta} f(z)=I^{\delta} f(z)$ was studied by Jung et al. [5].

Using the operator $L_{a}^{\delta}$, we now introduce the following classes.

Definition 1.1. One says that a function $f \in \mathcal{A}$ is in the class $\mathcal{S}_{a, \delta}(b, \beta)$ if

$$
\operatorname{Re}\left\{1+\frac{1}{b}\left(\frac{z\left(L_{a}^{\delta} f(z)\right)^{\prime}}{L_{a}^{\delta} f(z)}-1\right)\right\}>\beta,
$$

where $z \in \mathbb{U} ; a>0 ; \delta \geq 0 ; 0 \leq \beta<1 ; b \in \mathbb{C} \backslash\{0\}$.

Definition 1.2. One says that a function $f \in \mathcal{A}$ is in the class $\mathcal{C}_{a, \delta}(b, \beta)$ if

$$
\operatorname{Re}\left\{1+\frac{1}{b} \frac{z\left(L_{a}^{\delta} f(z)\right)^{\prime \prime}}{\left(L_{a}^{\delta} f(z)\right)^{\prime}}\right\}>\beta,
$$

where $z \in \mathbb{U} ; a>0 ; \delta \geq 0 ; 0 \leq \beta<1 ; b \in \mathbb{C} \backslash\{0\}$. 
International Journal of Mathematics and Mathematical Sciences

Note that

$$
f \in \mathcal{C}_{a, \delta}(b, \beta) \Leftrightarrow z f^{\prime} \in \mathcal{S}_{a, \delta}(b, \beta) .
$$

In particular, the classes

$$
\mathcal{S}_{a, \delta}(b, 0) \equiv \mathcal{S}_{a, \delta}(b), \quad \mathcal{C}_{a, \delta}(b, 0) \equiv \mathcal{C}_{a, \delta}(b)
$$

introduced by Bulut [6].

Making use of the Komatu integral operator $L_{a}^{\delta}$, we now introduce each of the following subclasses of analytic functions.

Definition 1.3. One denotes by $\mathcal{S}_{a, \delta}(\lambda, b, A, B)$ the class of functions $f \in \mathcal{A}$ satisfying

$$
1+\frac{1}{b}\left(\frac{z\left(\lambda z\left(L_{a}^{\delta} f(z)\right)^{\prime}+(1-\lambda) L_{a}^{\delta} f(z)\right)^{\prime}}{\lambda z\left(L_{a}^{\delta} f(z)\right)^{\prime}+(1-\lambda) L_{a}^{\delta} f(z)}-1\right) \prec \frac{1+A z}{1+B z}
$$

where $z \in \mathbb{U} ; a>0 ; \delta \geq 0 ;-1 \leq B<A \leq 1 ; 0 \leq \lambda \leq 1 ; b \in \mathbb{C} \backslash\{0\}$.

Definition 1.4. A function $f \in \mathcal{A}$ is said to be in the class $\mathbb{B}_{a, \delta}(\lambda, b, A, B, m ; u)$ if it satisfies the following non-homogenous Cauchy-Euler differential equation:

$$
\begin{aligned}
& z^{m} \frac{d^{m} w}{d z^{m}}+\left(\begin{array}{c}
m \\
1
\end{array}\right)(u+m-1) z^{m-1} \frac{d^{m-1} w}{d z^{m-1}}+\cdots+\left(\begin{array}{c}
m \\
m
\end{array}\right) w \prod_{j=0}^{m-1}(u+j)=g(z) \prod_{j=0}^{m-1}(u+j+1) \\
& \left(w=f(z) \in \mathcal{A} ; g \in \mathcal{S}_{a, \delta}(\lambda, b, A, B) ; m \in \mathbb{N}^{*} ; u \in(-1, \infty)\right) .
\end{aligned}
$$

Remark 1.5. If we set $\delta=0$ in the classes $\mathcal{S}_{a, \delta}(\lambda, b, A, B)$ and $B_{a, \delta}(\lambda, b, A, B, m ; u)$, then we have the classes

$$
\mathcal{S}(\lambda, b, A, B), \quad \mathcal{K}(\lambda, b, A, B, m ; u)
$$

introduced by Srivastava et al. [7], respectively.

If we take $A=1-2 \beta(0 \leq \beta<1)$ and $B=-1$ in the class $\mathcal{S}_{a, \delta}(\lambda, b, A, B)$, then we have a new class consisting of functions $f \in \mathcal{A}$ which satisfy the condition

$$
\operatorname{Re}\left\{1+\frac{1}{b}\left(\frac{z\left(\lambda z\left(L_{a}^{\delta} f(z)\right)^{\prime}+(1-\lambda) L_{a}^{\delta} f(z)\right)^{\prime}}{\lambda z\left(L_{a}^{\delta} f(z)\right)^{\prime}+(1-\lambda) L_{a}^{\delta} f(z)}-1\right)\right\}>\beta, \quad z \in \mathbb{U}
$$

We denote this class by $\mathcal{S}_{a, \delta}(\lambda, b, \beta)$. Also we denote by $\boldsymbol{B}_{a, \delta}(\lambda, b, \beta, m ; u)$ for corresponding class to $\boldsymbol{B}_{a, \delta}(\lambda, b, 1-2 \beta,-1, m ; u)$. 
Note that taking $\lambda=0$ and $\lambda=1$ for the class $\mathcal{S}_{a, \delta}(\lambda, b, \beta)$, we have the classes $\mathcal{S}_{a, \delta}(b, \beta)$ and $\mathcal{C}_{a, \delta}(b, \beta)$, respectively. In particular, the classes

$$
\mathcal{S}_{a, 0}(\lambda, b, \beta) \equiv \mathcal{S C}(b, \lambda, \beta), \quad \mathcal{B}_{a, 0}(\lambda, b, \beta, 2 ; u) \equiv \mathcal{B}(b, \lambda, \beta ; u)
$$

are studied by Altıntaş et al. [8].

In this work, by using the principle of subordination, we obtain coefficient bounds for functions in the subclasses

$$
\mathcal{S}_{a, \delta}(\lambda, b, A, B), \quad B_{a, \delta}(\lambda, b, A, B, m ; u)
$$

of analytic functions of complex order, which we have introduced here. Our results would unify and extend the corresponding results obtained earlier by Robertson [9], Nasr and Aouf [10], Altıntaş et al. [8] and Srivastava et al. [7].

In our investigation, we will make use of the principle of subordination between analytic functions, which is explained in Definition 1.6 below (see [11]).

Definition 1.6. For two functions $f$ and $g$, analytic in $\mathbb{U}$, one says that the function $f(z)$ is subordinate to $g(z)$ in $\mathbb{U}$, and write

$$
f(z) \prec g(z), \quad z \in \mathbb{U},
$$

if there exists a Schwarz function $w(z)$, analytic in $\mathbb{U}$, with

$$
w(0)=0, \quad|w(z)|<1, \quad z \in \mathbb{U},
$$

such that

$$
f(z)=g(w(z)), \quad z \in \mathbb{U} .
$$

In particular, if the function $g$ is univalent in $\mathbb{U}$, the above subordination is equivalent to

$$
f(0)=g(0), \quad f(\mathbb{U}) \subset g(\mathbb{U}) .
$$

In order to prove our main results (Theorems 2.1 and 2.2 in Section 2), we first recall the following lemma due to Rogosinski [12].

Lemma 1.7. Let the function $g$ given by

$$
g(z)=\sum_{k=1}^{\infty} b_{k} z^{k}, \quad z \in \mathbb{U}
$$


International Journal of Mathematics and Mathematical Sciences

be convex in $\mathbb{U}$. Also let the function $f$ given by

$$
f(z)=\sum_{k=1}^{\infty} a_{k} z^{k}, \quad z \in \mathbb{U}
$$

be holomorphic in $\mathbb{U}$. If

$$
f(z) \prec g(z), \quad z \in \mathbb{U},
$$

then

$$
\left|a_{k}\right| \leq\left|b_{1}\right|, \quad k \in \mathbb{N} .
$$

\section{The Main Results and Their Demonstration}

We now state and prove each of our main results given by Theorems 2.1 and 2.2 below.

Theorem 2.1. Let the function $f \in \mathcal{A}$ be defined by (1.3). If the function $f$ is in the class $\mathcal{S}_{a, \delta}(\lambda, b, A, B)$, then

$$
\left|a_{n}\right| \leq\left(\frac{a+n-1}{a}\right)^{\delta} \frac{\prod_{j=0}^{n-2}[j+|b|(A-B)]}{(n-1) !(1+\lambda(n-1))}, \quad n \in \mathbb{N}^{*}
$$

Proof. Let the function $f \in \mathscr{A}$ be given by (1.3). Define a function

$$
h(z)=\lambda z\left(L_{a}^{\delta} f(z)\right)^{\prime}+(1-\lambda) L_{a}^{\delta} f(z), \quad z \in \mathbb{U}
$$

We note that the function $h$ is of the form

$$
h(z)=z+\sum_{n=2}^{\infty} A_{n} z^{n}, \quad z \in \mathbb{U}
$$

where, for convenience,

$$
A_{n}=(1+\lambda(n-1))\left(\frac{a}{a+n-1}\right)^{\delta} a_{n}, \quad n \in \mathbb{N}^{*}
$$

From Definition 1.3 and (2.2), we obtain that

$$
1+\frac{1}{b}\left(\frac{z h^{\prime}(z)}{h(z)}-1\right) \prec \frac{1+A z}{1+B z}, \quad z \in \mathbb{U} .
$$


Let us set

$$
g(z)=\frac{1+A z}{1+B z}
$$

and define the function $p(z)$ by

$$
p(z)=1+\frac{1}{b}\left(\frac{z h^{\prime}(z)}{h(z)}-1\right), \quad z \in \mathbb{U}
$$

Therefore, we have

$$
p(z) \prec g(z), \quad z \in \mathbb{U} \text {. }
$$

Hence, by Definition 1.6, we deduce that

$$
p(z)=\frac{1+A w(z)}{1+B w(z)} \quad(w(0)=0 ;|w(z)|<1)
$$

Note that

$$
p(0)=g(0)=1, \quad p(z) \in g(\mathbb{U}), \quad z \in \mathbb{U}
$$

Also from (2.7), we find

$$
z h^{\prime}(z)=[1+b(p(z)-1)] h(z)
$$

Let

$$
p(z)=1+c_{1} z+c_{2} z^{2}+\cdots, z \in \mathbb{U}
$$

Since $A_{1}=1$, in view of (2.3), (2.11) and (2.12), we obtain

$$
(n-1) A_{n}=b\left\{c_{n-1}+c_{n-2} A_{2}+\cdots+c_{1} A_{n-1}\right\}
$$

for $n \in \mathbb{N}^{*}$. On the other hand, according to the Lemma 1.7, we obtain

$$
\left|\frac{p^{(m)}(0)}{m !}\right| \leq A-B, \quad m \in \mathbb{N}
$$


International Journal of Mathematics and Mathematical Sciences

By combining (2.14) and (2.13), for $n=2,3,4$, we obtain

$$
\begin{gathered}
\left|A_{2}\right| \leq|b|(A-B) \\
\left|A_{3}\right| \leq \frac{|b|(A-B)(1+|b|(A-B))}{2 !} \\
\left|A_{4}\right| \leq \frac{|b|(A-B)(1+|b|(A-B))(2+|b|(A-B))}{3 !}
\end{gathered}
$$

respectively. Using the principle of mathematical induction, we obtain

$$
\left|A_{n}\right| \leq \frac{\prod_{j=0}^{n-2}[j+|b|(A-B)]}{(n-1) !}, \quad n \in \mathbb{N}^{*} .
$$

Now from (2.4), it is clear that

$$
\left|a_{n}\right| \leq\left(\frac{a+n-1}{a}\right)^{\delta} \frac{\prod_{j=0}^{n-2}[j+|b|(A-B)]}{(n-1) !(1+\lambda(n-1))}, \quad n \in \mathbb{N}^{*} .
$$

This evidently completes the proof of Theorem 2.1.

Theorem 2.2. Let the function $f \in \mathcal{A}$ be defined by (1.3). If the function $f$ is in the class $B_{a, \delta}(\lambda, b, A, B, m ; u)$, then

$$
\left|a_{n}\right| \leq\left(\frac{a+n-1}{a}\right)^{\delta} \frac{\prod_{j=0}^{n-2}[j+|b|(A-B)]}{(n-1) !(1+\lambda(n-1))} \frac{\prod_{j=0}^{m-1}(u+j+1)}{\prod_{j=0}^{m-1}(u+j+n)}, \quad n \in \mathbb{N}^{*} .
$$

Proof. Let the function $f \in \mathcal{A}$ be given by (1.3). Also let

$$
q(z)=z+\sum_{n=2}^{\infty} B_{n} z^{n} \in \mathcal{S}_{a, \delta}(\lambda, b, A, B)
$$

so that

$$
a_{n}=\frac{\prod_{j=0}^{m-1}(u+j+1)}{\prod_{j=0}^{m-1}(u+j+n)} B_{n}, \quad n \in \mathbb{N}^{*}, \quad u \in(-1, \infty) .
$$

Thus, by using Theorem 2.1, we obtain

$$
\left|a_{n}\right| \leq\left(\frac{a+n-1}{a}\right)^{\delta} \frac{\prod_{j=0}^{n-2}[j+|b|(A-B)]}{(n-1) !(1+\lambda(n-1))} \frac{\prod_{j=0}^{m-1}(u+j+1)}{\prod_{j=0}^{m-1}(u+j+n)} .
$$

This completes the proof of Theorem 2.2. 


\section{Corollaries and Consequences}

In this section, we apply our main results (Theorems 2.1 and 2.2) in order to deduce each of the following corollaries and consequences.

It is easy to see that

$$
j+|b|(A-B) \leq j+\frac{2|b|(A-B)}{1-B}, \quad j \in \mathbb{N}^{*},-1 \leq B<A \leq 1, b \in \mathbb{C} \backslash\{0\},
$$

which would obviously yield significant improvements over Theorems 2.1 and 2.2 (see Srivastava et al. [7]).

Setting $A=1-2 \beta(0 \leq \beta<1)$ and $B=-1$ in Theorems 2.1 and 2.2, we have

Corollary 3.1. Let the function $f \in \mathcal{A}$ be defined by (1.3). If the function $f$ is in the class $\mathcal{S}_{a, \delta}(\lambda, b, \beta)$, then

$$
\left|a_{n}\right| \leq\left(\frac{a+n-1}{a}\right)^{\delta} \frac{\prod_{j=0}^{n-2}[j+2|b|(1-\beta)]}{(n-1) !(1+\lambda(n-1))}, \quad n \in \mathbb{N}^{*} .
$$

Remark 3.2. Taking $\delta=0$ in Corollary 3.1, we have [8, Theorem 1].

Corollary 3.3. Let the function $f \in \mathcal{A}$ be defined by (1.3). If the function $f$ is in the class $B_{a, \delta}(\lambda, b, \beta, m ; u)$, then

$$
\left|a_{n}\right| \leq\left(\frac{a+n-1}{a}\right)^{\delta} \frac{\prod_{j=0}^{n-2}[j+2|b|(1-\beta)]}{(n-1) !(1+\lambda(n-1))} \frac{\prod_{j=0}^{m-1}(u+j+1)}{\prod_{j=0}^{m-1}(u+j+n)}, \quad n \in \mathbb{N}^{*} .
$$

Remark 3.4. Taking $\delta=0$ and $m=2$ in Corollary 3.3, we have [8, Theorem 2].

Letting $\lambda=0$ and $\lambda=1$ in Corollary 3.1, we get following corollaries, respectively.

Corollary 3.5. Let the function $f \in \mathcal{A}$ be defined by (1.3). If the function $f$ is in the class $\mathcal{S}_{a, \delta}(b, \beta)$, then

$$
\left|a_{n}\right| \leq\left(\frac{a+n-1}{a}\right)^{\delta} \frac{\prod_{j=0}^{n-2}[j+2|b|(1-\beta)]}{(n-1) !}, \quad n \in \mathbb{N}^{*}
$$

Corollary 3.6. Let the function $f \in \mathcal{A}$ be defined by (1.3). If the function $f$ is in the class $\mathcal{C}_{a, \delta}(b, \beta)$, then

$$
\left|a_{n}\right| \leq\left(\frac{a+n-1}{a}\right)^{\delta} \frac{\prod_{j=0}^{n-2}[j+2|b|(1-\beta)]}{n !}, \quad n \in \mathbb{N}^{*}
$$

For other related results, see also $[9,10]$. 


\section{References}

[1] Y. Komatu, "On analytic prolongation of a family of operators," Mathematica (Cluj), vol. 32 (55), no. 2, pp. 141-145, 1990.

[2] T. M. Flett, "The dual of an inequality of Hardy and Littlewood and some related inequalities," Journal of Mathematical Analysis and Applications, vol. 38, pp. 746-765, 1972.

[3] G. S. Sălăgean, "Subclasses of univalent functions," in Complex Analysis-Fifth Romanian-Finnish Seminar, Part 1 (Bucharest, 1981), vol. 1013 of Lecture Notes in Mathematics, pp. 362-372, Springer, Berlin, Germany, 1983.

[4] B. A. Uralegaddi and C. Somanatha, "Certain classes of univalent functions," in Current Topics in Analytic Function Theory, pp. 371-374, World Scientific, River Edge, NJ, USA, 1992.

[5] I. B. Jung, Y. C. Kim, and H. M. Srivastava, "The Hardy space of analytic functions associated with certain one-parameter families of integral operators," Journal of Mathematical Analysis and Applications, vol. 176, no. 1, pp. 138-147, 1993.

[6] S. Bulut, "Fekete-Szegö problem for subclasses of analytic functions defined by Komatu integral operator," submitted.

[7] H. M. Srivastava, O. Altıntaş, and S. K. Serenbay, "Coefficient bounds for certain subclasses of starlike functions of complex order," Applied Mathematics Letters, vol. 24, no. 8, pp. 1359-1363, 2011.

[8] O. Altıntaş, H. Irmak, S. Owa, and H. M. Srivastava, “Coefficient bounds for some families of starlike and convex functions of complex order," Applied Mathematics Letters, vol. 20, no. 12, pp. 1218-1222, 2007.

[9] M. S. Robertson, "On the theory of univalent functions," Annals of Mathematics (2), vol. 37, no. 2, pp. 374-408, 1936.

[10] M. A. Nasr and M. K. Aouf, "Radius of convexity for the class of starlike functions of complex order," Bulletin of the Faculty of Science Assiut University A, vol. 12, no. 1, pp. 153-159, 1983.

[11] S. S. Miller and P. T. Mocanu, Differential Subordinations: Theory and Applications, vol. 225 of Monographs and Textbooks in Pure and Applied Mathematics, Marcel Dekker, New York, NY, USA, 2000.

[12] W. Rogosinski, "On the coefficients of subordinate functions," Proceedings of the London Mathematical Society (2), vol. 48, pp. 48-82, 1943. 


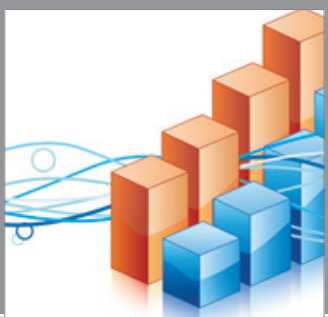

Advances in

Operations Research

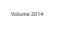

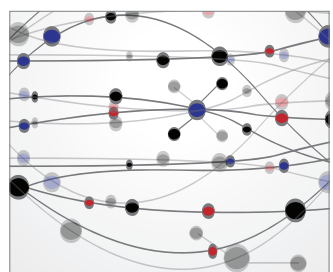

\section{The Scientific} World Journal
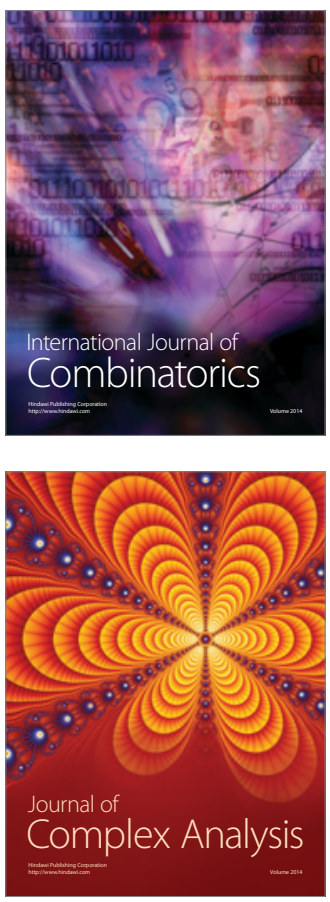

International Journal of

Mathematics and

Mathematical

Sciences
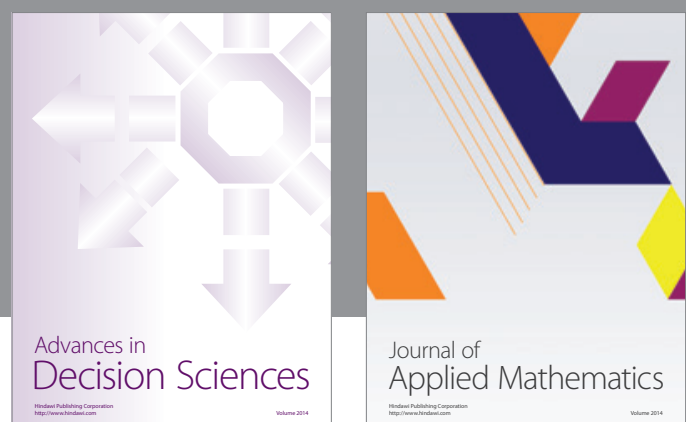

Journal of

Applied Mathematics
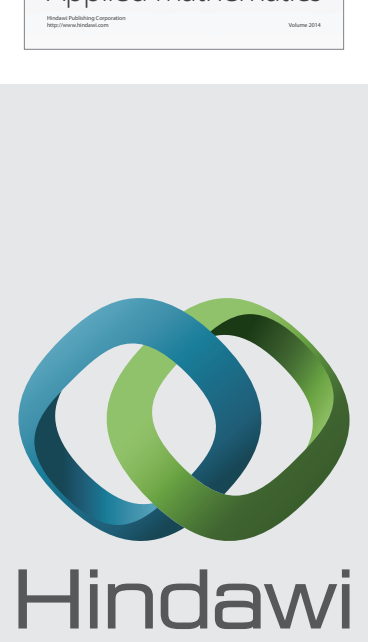

Submit your manuscripts at http://www.hindawi.com
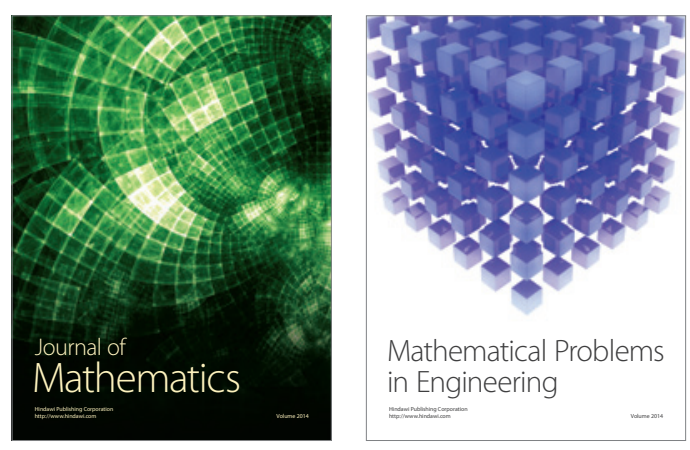

Mathematical Problems in Engineering
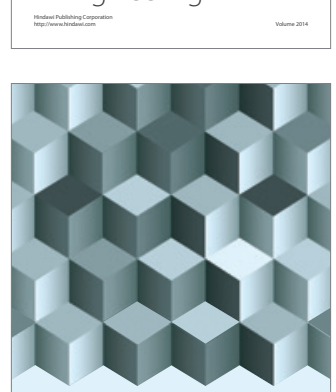

Journal of

Function Spaces
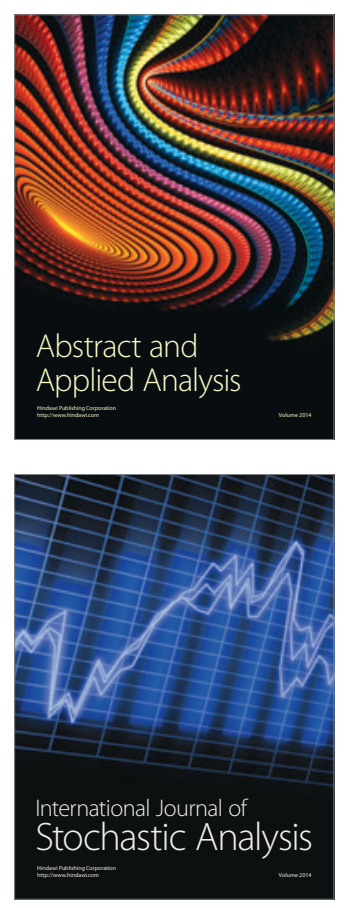

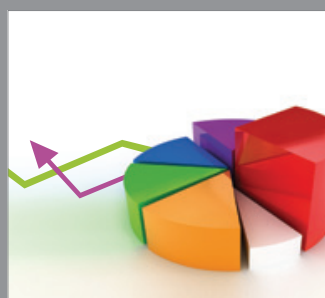

ournal of

Probability and Statistics

Promensencen
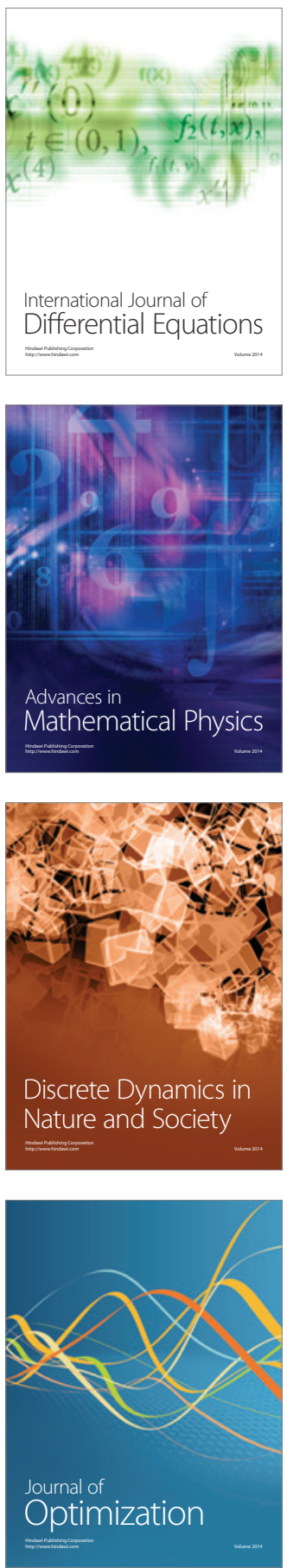